\title{
Real-Time PCR: an Appropriate Approach to Confirm ssDNA Generation from PCR Product in SELEX Process
}

\author{
Shirin Kouhpayeh ${ }^{1}$, Zahra Hejazi ${ }^{2}$, Hossein Khanahmad ${ }^{2,3}$, Abbas Rezaei ${ }^{1 *}$ \\ ${ }^{1}$ Department of Immunology, School of Medicine, Isfahan University of Medical Sciences, Isfahan, 313, Iran \\ ${ }^{2}$ Department of Genetics and Molecular Biology, School of Medicine, Isfahan University of Medical Sciences, Isfahan, 313 , Iran \\ ${ }^{3}$ Pediatric Inherited Diseases Research Center, Research Institute for Primordial Prevention of Non-communicable disease, Isfahan \\ University of Medical Sciences, Isfahan, 313, Iran
}

*Corresponding author: Abbas Rezaei, Department of Immunology, School of Medicine, Isfahan University of Medical Sciences, Isfahan, Iran. Phone/ Fax:+98-31-37922460, E-mail: rezaei@mui.ac.ir, rezaei1335@yahoo.com

Received: 7 April 2016; $\quad$ Revised: 9 May 2017; $\quad$ Accepted: 20 June 2017 ; $\quad$ Published online: 19 August 2017

Background: Aptamers are single stranded DNA (ssDNA) or RNA molecules. The potential of aptamers for binding to the different targets has made them be widely used as the preferred diagnostic and therapeutic tools. DNA aptamers present several advantages over the RNA oligonucleotides due to their higher stability, easier selection, and production. Selection of DNA aptamers which is facilitated through a systematic evolution of ligand by exponential enrichment (SELEX) method is much dependent on the successful conversion of double stranded DNA (dsDNA) to ssDNA.

Objective: There are different methods available for ssDNA generation. While visualization of ssDNA is limited to the gelbased method, the method is not applicable in the initial rounds of SELEX due to more than $10^{15}$ different sequences. This study was designed to evaluate the efficiency of another technique for confirming the ssDNA generation in comparison to the polyacrylamide electrophoresis (PAGE) analysis.

Materials and Methods: Real-time PCR was employed in the present study for PCR amplification of the initial library that was followed by enzymatic digestion of the dsDNA. Subsequently melting curve analysis was carried out to evaluate ssDNA generation from dsDNA. Moreover, PAGE analysis was performed and the results were compared with the melt curve analysis.

Results: The melt curves, revealed dsDNA conversion to the ssDNA based on a significant reduction of Tm from 73.8 to $41.5^{\circ} \mathrm{C}$. Applying PAGE analysis, it was not effectively feasible to show ssDNA generation from the corresponding initial dsDNA library, while, it was efficient enough to confirm ssDNA generation in accordance with the increasing the number of SELEX rounds.

Conclusion: The present study has proven the applicability of the real-time PCR as a suitable confirmatory technique for validating ssDNA generation in the DNA aptamer selection process for the initial library preparation.

Keywords: Half- Renaturation, Melt Curve, PAGE, Real Time PCR, SELEX.

\section{Background}

Aptamers are single stranded nucleic acid DNA (ssDNA) or RNA molecules which can bind to a wide range of targets including proteins, small molecules, and even viruses or the whole cells through their unique three-dimensional (3D) structures (1). Aptamers are qualified to be employed as diagnostic and therapeutic tools through their high specificity and affinity for the given targets (2). DNA aptamers are preferred in comparison to their RNA counterpart due to a number of advantages such as higher stability with respect to temperature, resistance to chemical hydrolysis, a higher feasibility in the selected process, enrichment process, and the lower production costs for the initial library preparation (3). The successful selection of DNA aptamers is performed through a PCR-based process called SELEX (systematic evolution of ligand by exponential enrichment) $(4,5)$.

SELEX is a sequential panning method where a large and random sequence library is exposed to a 
specific target. The bound sequences are then recovered and amplified by PCR (sub-library). The binding/ amplification phases are repeated to enrich the aptamer pool $(4,5)$. Isolation of the high-affinity aptamers against a specified target is dependent on the efficient production of ssDNA from the double stranded DNA (dsDNA) in subsequent to the amplification step by SELEX since the only ssDNA can form the distinctive conformation which is required for a target specific binding (6).

Several methods have been developed for the generation of ssDNA following to the PCR amplification including asymmetric PCR, accompanied by the production of ssDNA together with the dsDNA as a key problem $(3,7)$. Another convenient method is biotinstreptavidin separation, although reannealing of two biotin and streptavidin conjugated strands throughout the process is always proposed as a disadvantage (8). Another commonly used method for ssDNA generation is the application of lambda exonuclease III although it is costly. Size separations on denaturing urea polyacrylamide gel electrophoresis (PAGE) is the other method for ssDNA generation which is timeconsuming and costly due to using modified primers with hexaethylene glycol (HEGL) or ribose residues (3).

All the aforementioned methods require an assessment technique to evaluate and confirm the authenticity of the process which is mostly based on agarose or polyacrylamide gel electrophoresis.

In the preliminary rounds of the SELEX with an approximately $10^{15}$ or more different sequences as potential aptamers in the initial library $(9,10)$, using conventional electrophoretic methods to evaluate ssDNA production is not reliable due to the presence of various sequences with different conformations leading to the complicated results. Hence, the lack of an appropriate method for ssDNA verification in the early rounds of the SELEX, regarding verification of the generated ssDNA, it is necessary to employ another method rather than using conventional techniques to confirm ssDNA isolation (11).

\section{Objective}

Considering the SYBR green dye function upon binding to the double-stranded DNA by intercalation and/or minor groove binding (12), thus identification of ssDNA/dsDNA may be achieved by melting curve analysis using SYBR green dye.

The present study was designed to evaluate the role of real-time PCR and melt curve analysis for confirming ssDNA production from PCR product in the initial rounds of SELEX and comparing the efficiency of this technique with PAGE analysis.

\section{Materials and Methods}

\section{1. ssDNA Library and Primers}

The initial ssDNA library (5'-ATACCA GCTTATTCAATT-52N-AGATAGTAAGTGCA ATCT-3') consists of a randomized 52 nucleotide region, flanked by the two 18 nucleotide constant regions was synthesized by Tag Copenhagen company (Tag Copenhagen A/S, Denmark). The synthetic ssDNA library $(787 \mu \mathrm{g})$, was used as a template for quantitative real time PCR using ABI StepOnePlus (StepOnePlus Real time PCR, Life Technology, USA) applying different prepared dilutions : $1 / 4\left(33 \mathrm{ng} . \mu \mathrm{L}^{-1}\right), 1 / 20(6.7$ ng. $\left.\mu \mathrm{L}^{-1}\right)$, and $1 / 40\left(0.67\right.$ ng. $\left.\mu \mathrm{L}^{-1}\right)$ which were assayed in two set of triplicate samples.

\subsection{Real Time PCR}

The reaction mixture containing $10 \mu \mathrm{L}$ of SYBR green PCR master mix (Thermo Scientific, Waltham, MA, USA), $(0.5 \mathrm{nM})$ of the each forward and reverse primers, 5'-ATACCAGCTTATTCAATT-3' and 5'-PhosphateAGATTGCACTTACTATCT-3' respectively (Tag Copenhagen A/S, Denmark) was adjusted to a final volume of $20 \mu \mathrm{L}$, using deionized water.

Both sets of triplicate dilutions were heated to $95{ }^{\circ} \mathrm{C}$ for 10 minutes, followed by 6 cycles of $95^{\circ} \mathrm{C}$ for 30 sec, $42{ }^{\circ} \mathrm{C}$ for $30 \mathrm{sec}$, and $72{ }^{\circ} \mathrm{C}$ for $30 \mathrm{sec}$ and a final extension step that was conducted for $3 \mathrm{~min}$ at $72{ }^{\circ} \mathrm{C}$.

\subsection{Lambda Exonuclease Digestion}

The amplification process was followed by lambda exonuclease III (Thermo Scientific, Waltham, MA, USA) digestion using the lambda exonuclease reaction buffer for one set of the triplicate dilutions which was facilitated through using a phosphorylated 5"-end reverse primer, while the other two sets of the diluted triplicates were left untreated. The mixtures were incubated at $37^{\circ} \mathrm{C}$ for $30 \mathrm{~min}$, followed by additional 15 min incubation at $80^{\circ} \mathrm{C}$ to stop the enzymatic reaction.

\subsection{Melting Curve Analysis}

Melting curve analysis was performed to evaluate the ssDNA generation from PCR product. To obtain the profile of the melting curve, both the treated and untreated dilutions were incubated at $95{ }^{\circ} \mathrm{C}$ for $15 \mathrm{sec}$, subsequently were cooled down to $30^{\circ} \mathrm{C}$ for $1 \mathrm{~min}$, and followed by an additional heating up to $95{ }^{\circ} \mathrm{C}$ for $15 \mathrm{sec}$ which was facilitated by employing a slope of $0.3^{\circ} \mathrm{C} / \mathrm{min}$. Alterations in the fluorescence intensity were monitored 
constantly and the melting temperatures $(\mathrm{Tm})$ were calculated with the Step One Plus software version 2.2 (StepOnePlus Real- time PCR, Life Technology, USA).

\subsection{PCR Amplification of ssDNA Library}

All PCR reagents were purchased from Thermo Scientific (Waltham, MA, USA). 33, 6.7 and 0.67 ng. $\mu \mathrm{L}^{-1}$ of initial ssDNA library plus $11^{\text {th }}$ selected pool was first amplified in two sets of PCR reaction using C1000 thermal cycler Bio-Rad (Thermo Scientific, Waltham, MA, USA). The reaction mixture contained $5 \mu \mathrm{L}$ of $10 \mathrm{X}$ PCR buffer, $5 \mu \mathrm{L}$ of each samples (each concentration of library), $2 \mu \mathrm{L}(10 \mu \mathrm{M})$ of each forward (5'-ATACCAGCTTATTCAATT-3') and reverse primers (5'-Phosphate-AGATTGCACTTACTATCT-3'), 1.5 $\mu \mathrm{L}(50 \mathrm{mM}) \mathrm{MgCl}_{2}, 1 \mu \mathrm{L}$ of dNTP $(10 \mathrm{mM})$, and the volume was adjusted to $50 \mu \mathrm{L}$ with deionized water. PCR program was started with 1 cycle at $95{ }^{\circ} \mathrm{C}$ for 3 min, followed by 6 cycles of $95^{\circ} \mathrm{C}$ for $30 \mathrm{sec}, 42{ }^{\circ} \mathrm{C}$ for $30 \mathrm{sec}$, and $72{ }^{\circ} \mathrm{C}$ for $30 \mathrm{sec}$. A final extension step was conducted for $3 \mathrm{~min}$ at $72^{\circ} \mathrm{C}$. One set of PCR products then treated with Lambda exonuclease III (Thermo Scientific, Waltham, MA, USA) in order to ssDNA generation as described above.

\subsection{Polyacrylamide Gel Electrophoresis (PAGE)}

Samples from the initial library as well as the $11^{\text {th }}$ selected pool, were loaded on a $12 \%$ non-denaturing PAGE in $1 \%$ TBE buffer following to heat denaturation at $95{ }^{\circ} \mathrm{C}$ for $5 \mathrm{~min}$ and subsequent snap-cooling on the ice. The electrophoresis was carried out at $4{ }^{\circ} \mathrm{C}$ using $70 \mathrm{~V}$ for $2 \mathrm{~h}$ following to a pre-running of the gel for 10 min at $90 \mathrm{~V}$. The gel was stained with silver-nitrate for visualization (1).

\subsection{Statistical Analysis}

The independent sample t-test was used for statistical analysis to compare the Tm of exonuclease lambda treated PCR product with that of untreated one. The comparison of the sample concentration and the required time for half- renaturation was also evaluated using independent sample t-test. P values less than 0.05 were considered as statistically significant. Statistical analysis was done using software package SPSS version 17.0 (SPSS Inc., Chicago, IL, USA). The results were presented as mean \pm standard deviation (SD).

\section{Results}

\subsection{Melting Curve Analysis}

The obtained results illustrated a significant reduction in Tm upon PCR product digestion with lambda exonuclease III $(43.69 \pm 1.08)$ in comparison to the untreated PCR product $(75.29 \pm 1.23)$ ( $\mathrm{P}$ value < 0.001 ) for $33 \mathrm{ng} . \mu \mathrm{L}^{-1}$ of each sample. A significant difference was also observed when Tm of 6.7 and $0.67 \mathrm{ng} . \mu \mathrm{L}^{-1}$ treated samples $(40.72 \pm 0.92$ and $40.33 \pm$ 1.13 , respectively) were compared with an equivalent untreated sample $(73.50 \pm 0.86$ and $72.74 \pm 0.58$, accordingly), ( $\mathrm{P}$ value $<0.001$ and the $\mathrm{P}$ value $<0.001$, respectively). The data are presented in Figure 1.

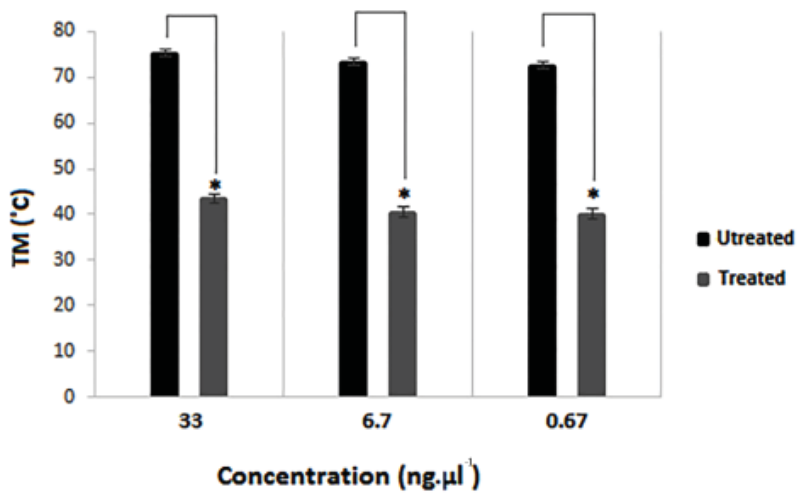

Figure 1. A representative comparison between the treated samples with their corresponding untreated samples Tm based on library concentration. The treated samples showed significantly lower Tm compared to the same concentrations of untreated samples $* \mathrm{P}<0.001$.

The results of the present study have demonstrated that the required time for half renaturation was significantly reduced in the case of treated sample (45.58 $\pm 3.69 \mathrm{~min}$ ) when compared to an equivalent untreated sample $(150.97 \pm 4.11 \mathrm{~min}, \mathrm{P}$ value $<0.001)$ for 33 ng. $\mu \mathrm{L}^{-1}$ of initial library. The same significant result was also observed for comparison of treated (35.74 \pm 3.09 and $34.40 \pm 3.81 \mathrm{~min})$ with untreated $(144.99 \pm$ 2.90 and $142.45 \pm 1.96 \mathrm{~min})$ in 6.7 and $0.67 \mathrm{ng} . \mu \mathrm{L}^{-1}$ of the initial library ( $\mathrm{P}$ value $<0.001$ and the $\mathrm{P}$ value $<0.001$, respectively). Moreover, when a comparison was carried out for the half-renaturation required time between 33 ng. $\mu \mathrm{L}^{-1}$ of the treated sample with the lower concentrations of the 6.7 and $0.67 \mathrm{ng} . \mu \mathrm{L}^{-1}$, has also shown to be significant $(\mathrm{P}<0.001$ and $\mathrm{P}<0.001)$, respectively. The same significant results were observed when the required time for half-renaturation of $33 \mathrm{ng} . \mu \mathrm{L}^{-1}$ untreated sample was compared to the 6.7 $(\mathrm{P}=0.003)$ and $0.67 \mathrm{ng} . \mu \mathrm{L}^{-1}(\mathrm{P}$ value $<0.001)$ of the untreated samples, respectively. While the difference of the required time for the half-renaturation between 6.7 and $0.67 . \mu \mathrm{L}^{-1}$ in treated or untreated samples was not significant $(\mathrm{P}=0.43$ and $\mathrm{P}=0.05$, respectively). These comparative data are pictorially presented in Figure 2. 


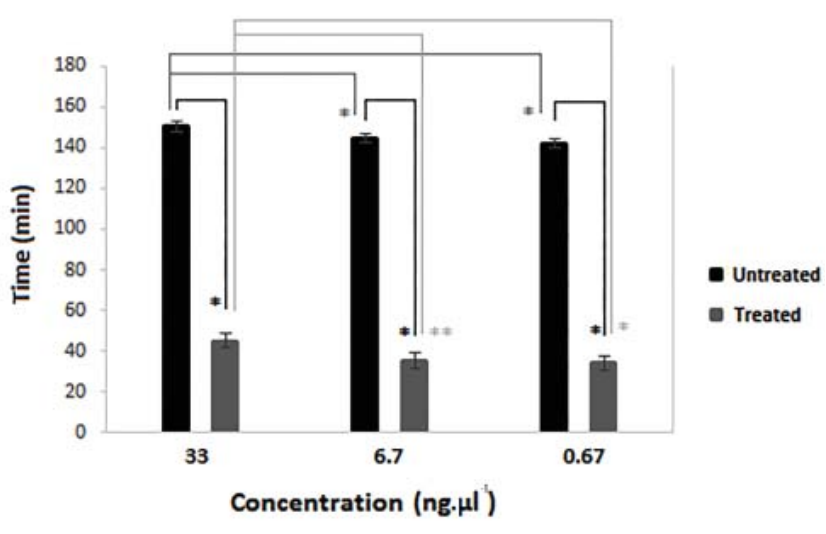

Figure 2. The collective comparison between the treated and untreated samples with respect to the required time for halfrenaturation based on the library concentration. The overall time for untreated samples was significantly higher than treated dilutions $* \mathrm{P}<0.001$. The required time for half-renaturation of $33 \mathrm{ng} . \mu \mathrm{L}^{-1}$ of the treated sample was significantly higher when compared to the lower concentrations $(* \mathrm{P}<0.001)$. The half-renaturation time for $33 \mathrm{ng} . \mu \mathrm{L}^{-1}$ of the untreated sample was also significant when compared to the untreated 6.7 and $0.67 \mathrm{ng} . \mu \mathrm{L}^{-1}$ concentrations $(* * \mathrm{P}<0.05$ and $* \mathrm{P}<$ $0.001)$, respectively.

\subsection{Polyacrylamide Gel Electrophoresis}

The result of the non-denaturing PAGE showed no distinction between the different dilutions of the ssDNA library PCR products and the corresponding treated samples, while, PCR product on $11^{\text {th }}$ selected pool and the lambda exonuclease treated samples of this round, were noticeably detectable in the same gel condition (Figs. 3 and 4, respectively).

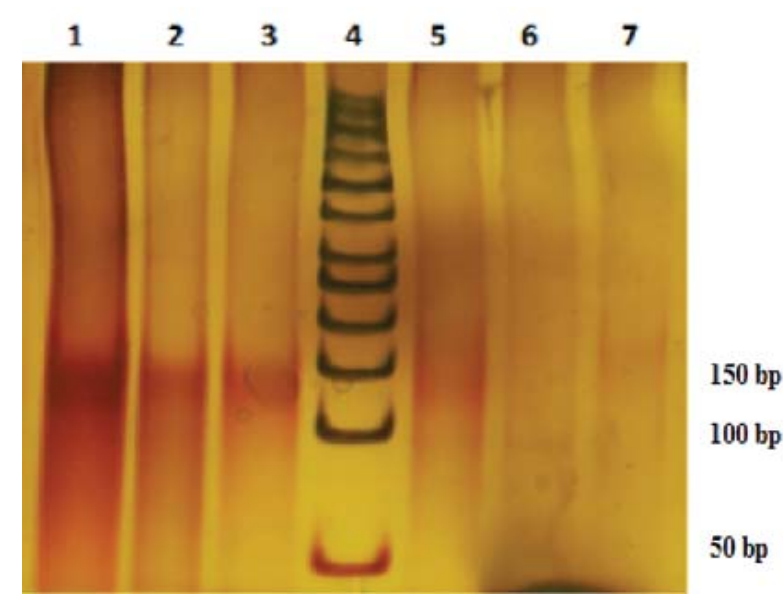

Figure 3. A representative PAGE analysis of the lambda exonuclease III treated in parallel with an untreated initial library samples. Lane 1-3: the initial library PCR product $\left(33,6.7\right.$, and $0.67 \mathrm{ng} . \mu \mathrm{L}^{-1}$, respectively), lane 4: DNA ladder (50 bp), lane 5-7: the initial library PCR product treated with lambda exonuclease $\left(33,6.7\right.$, and $0.67 \mathrm{ng} . \mu \mathrm{L}^{-1}$, respectively).

\section{Discussion}

Conventional methods for visualization and verification of the ssDNA generation from the PCR product seems inadequate for the initial rounds of the aptamer SELEX due to the presence of more than $10^{15}$ different sequences in the preliminary library. As was mentioned before, verification of ssDNA generation is a critical step in SELEX process due to the ability of ssDNA to form proper 3D structure to bind the target of interest with a high affinity and specificity (5). Regarding the recent problem in the aptamer selection in the initial rounds of the SELEX, coupled with the ability to discriminate ssDNA and dsDNA through corresponding Tm of such strands in the presence of an intercalating dye, the idea of using real-time PCR and melt-curve analysis was proposed.

In the present study, the melting temperatures of the lambda exonuclease treated PCR products of the initial library in different concentrations was compared to the equivalent dsDNA samples which were left untreated.

The results obtained from the melting curve behavior in the present study revealed that the melting temperature of the lambda exonuclease treated PCR products seem statistically reduced in comparison to the PCR products without exonuclease treatment. Reduction in the melting temperature upon treatment is attributed to be due to the degradation of the phosphorylated strand of the PCR amplified library from 5' to $3^{\prime}$ through lambda exonuclease activity as an exodeoxyribonuclease. The ssDNA only contains the secondary structure with weak interactions and needs a lower temperature to unfold $\left(45^{\circ} \mathrm{C}\right)$ in contrast to the dsDNA which needs a higher

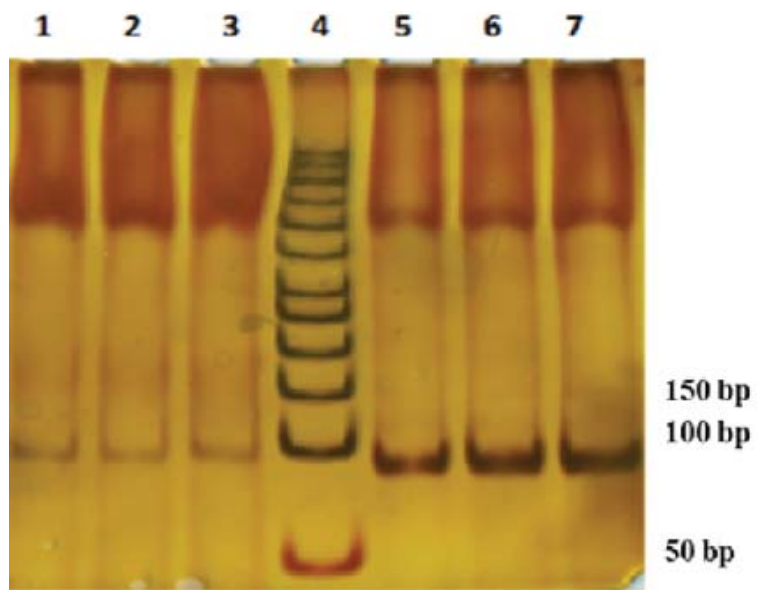

Figure 4. The PAGE analysis of the samples treated with the lambda exonuclease III and untreated 11th selected pool. Lanes 1-3: the 11th selected pool PCR product (33, 6.7, and $0.67 \mathrm{ng} . \mu \mathrm{L}^{-1}$ ), lane 4: the ladder (DNA, $50 \mathrm{bp}$ ), and lanes 5-7: the 11th selected pool PCR product of the exonuclease III treated samples (33, 6.7, and $0.67 \mathrm{ng} . \mu \mathrm{L}^{-1}$, respectively). 
temperature for denaturation $\left(73^{\circ} \mathrm{C}\right)(13)$.

Melting curve analysis is based on measuring the fluorescence intensity of all samples through the presence of SYBR Green as an intercalating dye. Since SYBR Green is known as a dsDNA binding dye, the fluorescence intensity increases for dsDNA or decreases upon the separation of the two strands from each other. The assay is based on heating up the lambda exonuclease treated and untreated samples in the presence of SYBR Green as an intercalating dye. Thus when temperature is raised to the melting temperature $\left(95^{\circ} \mathrm{C}\right)$, the PCR product denature and the DNA strands start to separate and release SYBR Green dye, resulting in the decline of the fluorescence intensity, while, in re-annealing temperature, the two strands could zip up again which lead to an increased fluorescence intensity (14).

The present data have illustrated that, as the degree of dilution increases less complexity is achieved and lower amounts of different sequences are retrieved, for which a shorter time for half-renaturation is required according to the equation $\mathrm{C} 0 \mathrm{t} 1 / 2 \alpha \mathrm{N}$, where, $\mathrm{N}$ is the complexity of sequences and $\mathrm{C} 0 \mathrm{t} 1 / 2$ is the required time for half-renaturation (15). It could be concluded that less complexity in the more diluted samples is directly proportional with the half-renaturation, although, calculation of the $\mathrm{N}$ is almost impossible due to the presence of more than $10^{12}$ oligonucleotides in the library (14).

Generally, with an increase in the dilution, fewer sequences are present in the reaction mixture, thus, the amplification process results in the more or less duplication of a limited number of sequences compared to the undiluted samples. Subsequently, re-melting analysis results in the sharper peaks with a larger area under the peak. This is due to the presence of less different sequences with more frequency after amplification.

As a confirmatory analysis in our present study, PAGE has shown the inadequacy of this technique for the initial rounds of SELEX due to the presence of practically $10^{15}$ different sequences which were presented as the smear in corresponding lanes for each sequence. Although with the progression of SELEX and exclusion of many sequences from the selection process, as shown for $11^{\text {th }}$ round, the limited number of selected sequences could be detected as separate bands through a non-denaturing PAGE. Therefore, PAGE analysis could be further employed for pool evolution monitoring through initial SELEX rounds. A recent study which evaluated different methods for SELEX progress monitoring also is in good accordance with our presented data (1).
Using Real-time PCR has previously been described in the SELEX process to monitor the SELEX progression or detection of the aptamer-target complexes (15). For the first time, our study has shown that Real-time PCR may serve as a reliable method for visualization of ssDNA generation from the PCR amplified product, particularly in the initial rounds of SELEX, with a higher number of different sequences which make the convenient methods of visualization, ineffective.

Conflict of interest statement: None declared.

Acknowledgments: This work was supported by grant No. 392607 from Isfahan University of Medical Sciences. We would also like to thank Dr. Laleh Shariati for her kind collaboration.

\section{References}

1. Mencin N, Šmuc T, Vraničar M, Mavri J, Hren M, Galeša K, et al. Optimization of SELEX: Comparison of different methods for monitoring the progress of in vitro selection of aptamers. J Pharm Biomed Anal. 2014;91:151-9. DOI: 10.1016/j. jpba.2013.12.031.

2. Sun H, Zhu X, Lu PY, Rosato RR, Tan W, Zu Y. Oligonucleotide aptamers: New tools for targeted cancer therapy. Mol Ther Nucleic Acids. 2014;3(8):e182. DOI: 10.1038/mtna.2014.32.

3. Marimuthu C, Tang T-H, Tominaga J, Tan S-C, Gopinath SC. Single-stranded DNA (ssDNA) production in DNA aptamer generation. Analyst. 2012;137(6):1307-15. DOI: 10.1039/ c2an15905h.

4. Orava EW, Cicmil N, Gariépy J. Delivering cargoes into cancer cells using DNA aptamers targeting internalized surface portals. Biochimica et Biophysica Acta. (BBA)Biomembranes. 2010;1798(12):2190-200. DOI: 10.1016/j. bbamem.2010.02.004.

5. Sefah K, Shangguan D, Xiong X, O'Donoghue MB, Tan W. Development of DNA aptamers using Cell-SELEX. Nat Protoc. 2010;5(6):1169-85. DOI: 10.1038/nprot.2010.66.

6. Liang $\mathrm{C}$, Li D, Zhang $\mathrm{G}, \mathrm{Li} \mathrm{H}$, Shao $\mathrm{N}$, Liang Z, et al. Comparison of the methods for generating single-stranded DNA in SELEX. Analyst. 2015;140(10):3439-44. DOI: 10.1007/ s00216-012-6183-4.

7. Svobodova M, Pinto A, Nadal P, O'Sullivan C. Comparison of different methods for generation of single-stranded DNA for SELEX processes. Anal Bioanal Chem. 2012;404(3):835-42. doi: 10.1007/s00216-012-6183-4.

8. Holmberg A, Blomstergren A, Nord O, Lukacs M, Lundeberg J, Uhlen M. The biotin-streptavidin interaction can be reversibly broken using water at elevated temperatures. Electrophoresis. 2005;26(3):501-10.

9. Kim YS, Gu MB. Advances in aptamer screening and small molecule aptasensors In: Biosensors Based on Aptamers and Enzymes: Springer; 2013.p. 29-67. DOI: 10.1007/10 2013225.

10. Pan W, Clawson GA. The shorter the better: reducing fixed primer regions of oligonucleotide libraries for aptamer selection. Molecules. 2009;14(4):1353-69. DOI: 10.3390/ molecules14041353. 
Kouhpayeh Sh. et al.

11. Avci-Adali M, Paul A, Wilhelm N, Ziemer G, Wendel HP. Upgrading SELEX technology by using lambda exonuclease digestion for single-stranded DNA generation. Molecules. 2009;15(1):1-11. DOI: 10.3390/molecules 15010001.

12. Varga A, James D. Real-time RT-PCR and SYBR Green I melting curve analysis for the identification of Plum pox virus strains $\mathrm{C}, \mathrm{EA}$, and $\mathrm{W}$ : effect of amplicon size, melt rate, and dye translocation. J Virol Methods. 2006;132(1):146-53. DOI: 10.1016/j.jviromet.2005.10.004

13. Dumousseau M, Rodriguez N, Juty N, Le Novere N. MELTING, a flexible platform to predict the melting temperatures of nucleic acids. BMC bioinformatics. 2012;13(1):1. DOI: 10.1186/14712105-13-101.

14. Schütze T, Arndt PF, Menger M, Wochner A, Vingron M, Erdmann VA, et al. A calibrated diversity assay for nucleic acid libraries using DiStRO-a Diversity Standard of Random Oligonucleotides. Nucleic Acids Res. 2009:gkp1108. DOI: 10.1093/nar/gkp1108

15. Vanbrabant J, Leirs K, Vanschoenbeek K, Lammertyn J, Michiels L. reMelting curve analysis as a tool for enrichment monitoring in the SELEX process. Analyst. 2014;139(3):58995. DOI: 10.1039/c3an01884a. 